\title{
Academia Chilena de Medicina
}

\section{Semblanza del Profesor Dr. Hernán Alessandri R.}

\author{
Dr. Alejandro Goic $\mathbf{G}$. \\ Biographical notes on Professor Hernan \\ Alessandri, M.D.
}

Hernán Alessandri, a renowned Chilean medical educator, was born in Santiago in 1900. He received his medical degree at the University of Chile in 1923. When in 1927 his father, then President of Chile, was sent into exile, he used the opportunity to deepen his medical knowledge in France and Germany. At the University of Chile, he became successively Professor of Clinical Medicine (1932), of Medical Semiology (1937), and Full Professor and Chair of Medicine (1944). At the Hospital del Salvador in Santiago, he organized a Clinical Department exemplary for its discipline, academic environment and dedication to patients and students. He was one of the prime movers for the reform of medical teaching in 1943, created medical residency programs for the training of specialists in 1952, served as Dean of the Faculty of Medicine from 1958 to 1962, and was a founding member of the Chilean Academy of Medicine (1964). He was the first Latin American to be named Honorary Member of the American College of Physicians (1968) and became Emeritus Professor of the University of Chile in 1973. He died in his hometown in 1982. His disciples and friends established in his honor a social and teaching foundation which they named after him. His clinical and diagnostic skills, along with his outstanding intelligence, made him the most brilliant clinician of his time and an exceptional medical educator who has inspired several generations of physicians (Rev Méd 2005; 133: 1500-3).

(Key Words: Education, medical; Famous persons; History of Medicine, 20th Century)

$\mathrm{H}$ istónicamente, el sentido originario $\mathrm{y}$, diná yo, más auténtico de ser médico es cuidar de los enfermos, es decir, ser un médico clínico. Lo clínico, en nigor, es todo lo que nos pone en contacto con la realidad individual de un enfermo y nos sirve para diagnosticar su enfermedad y tratarlo. Incluye, por consiguiente, tanto la exploración con los sentidos como la indagación psicológica y social y la exploración instrumental y de laboratorio. El arte de la medicina consiste, precisamente, en el conjunto de actos que, en su condición de clínico, realiza el médico: entrevistar, examinar, diagnosticar, pronosticar, tratar y educar a sus pacientes. El desarmollo experto de estas acciones médicas requiere relativamente más de habilidades intelectuales y sensoriales que de ciencia, en sentido estricto. En el ejercicio de la medicina clínica

1Presidente de la Academia Chilena de Medicina. Presentado en la XXXI Conferencia Anual Dr. Hernán Alessandri Rodríguez, el 19 de agosto de 2005, Auditorio del Hospital del Trabajador, ACHS, Santiago de Chile. todo es arte, es decir, habilidad sustentada en sus elementos esenciales: conocimientos, habilidades y destrezas, enriquecidas por la experiencia y la intuición, aplicadas en un ambiente de incertidumbre. El médico, aprovecha los conocimientos científicos para ejercer su arte clínico, lo que exige un complejo proceso intelectual de síntesis. Pero, no está de más recalcarlo, ni la ciencia ni la tecnología reemplazan el arte de la medicina, hoy tan amenazado por el cientificismo, la tecnología y el mercantilismo.

El gran clínico y profesor universitario que hoy recordamos: el Dr. Hemán Alessandri Rodríguez, fue un paradigma de una medicina ejencida como arte y ciencia, las dos vertientes complementarias de la práctica médica. Sus habilidades clínicas, semiológicas y diagnósticas, unidas a una preclara inteligencia, lo convirtieron en un eminente clínico - tal vez el más brillante de sus contemporáneos- y en un educador médico inigualado, que inspiró a varias generaciones de médicos y estudiantes que lo recuerdan hasta hoy con afecto y admiración, honrando el precepto 
hipocrático: ‘ener al que me enseñó este arte en igual estima que a mis progenitores».

Hernán Alessandri nació en Santiago en el año 1900 y se recibió de Médico-Cirujano en la Universidad de Chile, en 1923. Siendo estudiante, ingresó a la Asistencia Pública de Santiago, que en aquella época era el principal centro de atenciones de urgencias médicas y quinúrgicas del país, estación obligada de los jóvenes médicos que, estrictamente seleccionados, encontraban allí un lugar privilegiado de servicio, aprendizaje y perfeccionamiento profesional. De hecho, una brillante generación de grandes profesores de medicina de la Universidad de Chile, principalmente internistas, cirujanos y obstetras, pertenecieron a esa institución asistencial en las etapas iniciales de su carrera profesional. Destacado estudiante, Alessandri fue galardonado con el «remio Clin»que, en la época, se otorgaba a los mejores egresados de la Escuela de Medicina. Inició su vida académica en el Hospital San Vicente de Paul que, desde 1929 y hasta su desaparición en 1948, fue el hospital clínico de la Universidad de Chile, antecesor del Hospital José Joaquín Aguime inaugurado en 1953. Primero fue interno y, luego, al recibirse de médico, ayudante de la Cátedra de Clínica Médica del Profesor Daniel García Guemero quien, a juicio de Hernán Alessandri (que no era muy proclive a los halagos) era un clínico excepcionalmente talentoso. Fue, además, ayudante de la Cátedra de Zoología Médica y, luego, de Histología, del eminente Profesor Juan Noé, una figura señera de la medicina nacional, creador de varias disciplinas biológicas y a quien el país debe la erradicación de la malaria, que era endémica en la zona de Arica. Hijo del Presidente de la República don Arturo Alessandri Palma, durante un turbulento peńodo político fue deportado, en 1927, junto a sus padres y hermanos por el General Carlos Ibáñez del Campo. Ese acontecimiento lamentable, le permitió perfeccionar sus conocimientos al tomar contacto con la medicina y los profesores de Francia y Alemania, países que en la época estaban a la vanguardia del saber médico universal. De regreso a Chile, en 1929, se reincorporó al Hospital San Vicente de Paul, en la Cátedra del Dr. Ernesto Prado Tagle, pero lo abandonó a fines de 1930, por desavenencias con el profesor.

Ingresó, entonces, como médico ad-honorem al Servicio de Medicina del Hospital del Salvador de Santiago, en 1931, donde en los años siguientes iniciaría una profunda renovación de la enseñanza de la medicina y la organización de la atención

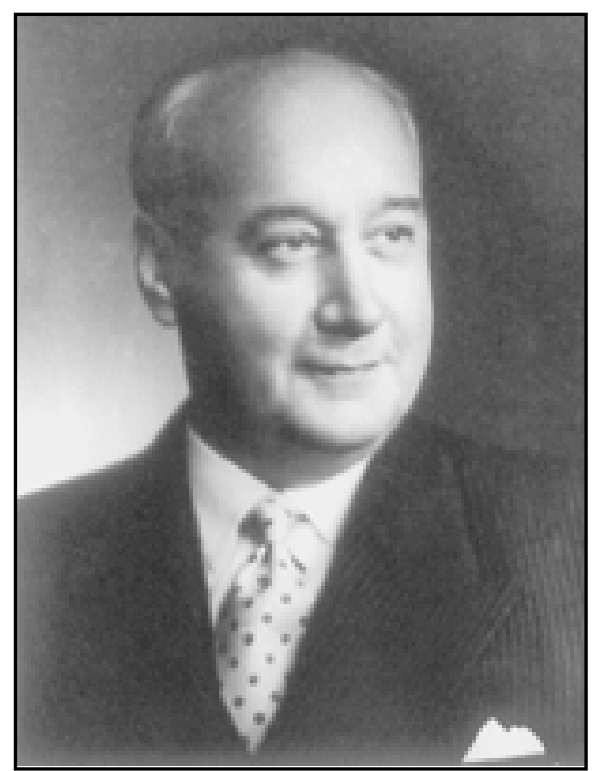

Dr. Hernán Alessandri R, en 1963.

médica hospitalaria. En 1932, la Universidad de Chile lo designó Profesor Extraordinario de Patología Médica, en 1937 la Facultad de Medicina lo eligió Profesor de Semiología Médica y, en 1944, Profesor Titular de la Cátedra « ¿»de Medicina. En su Cátedra, la enseñanza y práctica de la Semiología tuvo una gran relevancia, en la convicción de que esta disciplina no era la gramática de la medicina sino que la medicina misma». En el Hospital del Salvador, una reliquia de la arquitectura hospitalaria del siglo XIX que hoy está en riesgo de desaparecer, organizó un Servicio de Medicina y una Cátedra Universitaria ejemplares por su disciplina, ambiente académico y dedicación a los enfermos y a los estudiantes. Fue el líder indiscutido de un grupo de colaboradores directos: los Drs. Pedro García Palazuelos, Alejandro Olivares Concha, Héctor Ducci Claro, Eliseo Concha Parot, Mario Soza Werth, Raúl Etcheverry Barucchi, Eduardo Fritis Campusano, Arturo Atria Ramírez, Jacobo Lerner, Renato Gazmuri Ojeda, Werner Roeschmann von Bischoffhausen, Juan Zañartu, Lucía Toriello, Oke France Soto y, temporalmente, Luis Vargas Fernández, Amador Neghme Rodríguez y Jorge Torreblanca. Un conjunto de otros distinguidos médicos le acompañaron como tutores en la formación de los estudiantes de medicina: Gastón Chamorro Zapata, Nahum Sinay, Manuel Losada Losada, Fernando Cox Lira, David Lamas Brunstein, Gastón Dussaillant Grossetete, Ramón Florenzano 


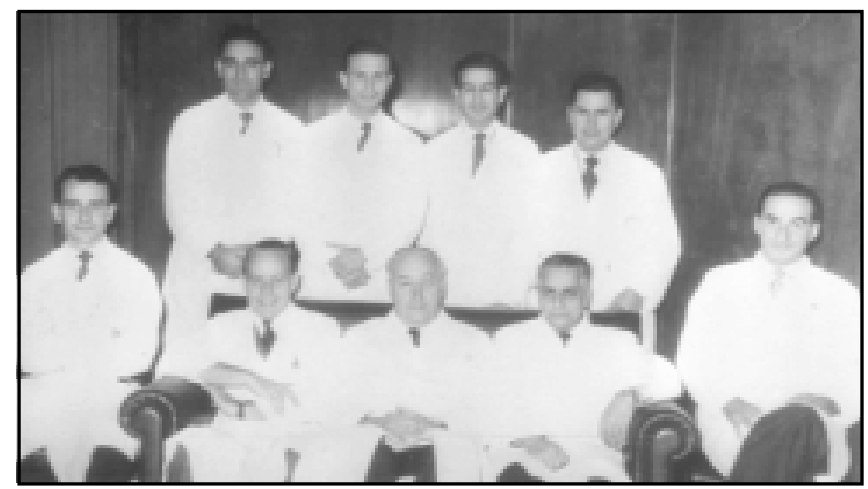

El Dr. Hernán Alessandri, en 1958, junto a los Drs. Héctor Ducci (izquierda) y Pedro García Palazuelos (derecha) y los becarios Drs. Alejandro Goic, Azaría Dueñas, Eduardo Mai, Gustavo D’Acuña, Juan Durán y Hernán Noguera.

González, Arturo Tamargo, Eduardo Díaz Carrasco, Jorge Palma Cereceda, José Barzelatto, Carlos Stevenson Rioseco, Hugo Donoso Puelma y Ricardo Katz Ugarte. En la década de los 50, se fueron incorporando a su Cátedra nuevas generaciones de destacados médicos que habían sido sus alumnos. El Dr. Héctor Ducci Claro, prematuramente fallecido, fue el colaborador más cercano del Profesor Alessandri y gracias a su dinamismo e innatas condiciones de administrador, le dio al Servicio de Medicina una organización y funcionamiento técnico, médico y de enfermería, realmente ejemplares. Alessandri estimuló la carrera profesional y académica de sus ayudantes, iniciando con ellos el desarrollo de las subespecialidades de la Medicina Interna en el país. Gran impulsor de las reuniones clínicas y anátomoclínicas, concebidas como fuentes irreemplazables de aprendizaje y perfeccionamiento profesional, las revistió de rigor y solemnidad, convirtiéndolas en una herramienta docente privilegiada, en las que participaban activamente internos, residentes y especialistas. Tuvo un importante papel en la Reforma de los Estudios Médicos de 1943, que introdujo la práctica clínica temprana de los estudiantes y diversificó los campos clínicos; en la creación de los programas formales de especialización médica, bajo el modelo estadounidense de Residencias hospitalarias, y de la Escuela de Graduados de la Facultad de Medicina, en 1954. Fue, precisamente, en su Servicio de Medicina donde se iniciaron en 1952 las primeras Residencias para la formación de especialistas en el país, años antes de su instauración formal por el Servicio Nacional de Salud y la Facultad de Medici- na. En reconocimiento de sus cualidades académicas y liderazgo fue elegido por sus pares Decano de la Facultad en 1958, cargo que ocupó hasta 1963, imprimiéndole su singular sello académico y acrecentando el desarrollo y prestigio institucional, con la colaboración de su amigo el Dr. Amador Neghme que le acompañó como Secretario de Facultad, cargo equivalente al actual de Vicedecano. Poco después del término de su Decanato, se acogió a jubilación, pero continuó concurriendo al hospital durante varios años más, atendiendo a los enfermos y entregando a los jóvenes estudiantes sus vastos conocimientos, experiencia y sabiduría. En 1964, al fundarse la Academia de Medicina y de acuerdo con la ley de creación del Instituto de Chile, fue propuesto por el Consejo Universitario de la Universidad de Chile como uno de los cinco Miembros de Número Fundadores de la corporación. Fue un privilegio haber estado presente en la solemne ceremonia realizada en Boston en 1968 en la que Hernán Alessandri fue distinguido por el American College of Physicians como Miembro Honora5S.S. rio de esa prestigiosa institución, siendo el primer médico latinoamericano que recibió tan alta distinción. En un discurso formal, agradeció a las instituciones médicas y fundaciones estadounidenses la valiosa ayuda prestada al progreso de la educación médica chilena. La Facultad de Medicina lo distinguió como Miembro Académico, en 1967, y la Universidad de Chile, como Profesor Emérito en 1973. En los últimos años de su vida y por aquellos inescrutables designios del destino, la luz de su brillante inteligencia, que había iluminado a la medicina nacional durante décadas, se fue apagando dolorosa y paulatinamente, falleciendo en Santiago en 1980 después de una prolongada enfermedad.

El Dr. Alessandri es reconocido como uno de los más eminentes clínicos del siglo XX y un connotado educador médico. Sus colaboradores, discípulos, amigos y otras personalidades públicas, de Chile y del extranjero, crearon en su memoria la «undación Social y Educativa Doctor Hemán Alessandri Rodríguez» para prolongar en el tiempo los caminos de su inspiración académica. Además, en reconocimiento y recuerdo de esta figura excepcional de la medicina chilena, sus conciudadanos le rindieron homenaje nominando con su nombre un 
Consultorio Externo de Salud, en la Municipalidad de Providencia, y una calle de la misma Comuna, cercana al hospital de sus afanes.

Con todo, y como suele ocurrir, su biografía no refleja en plenitud los matices y la riqueza de su personalidad y de su obra. Era un hombre relativamente alto, más bien corpulento, de frente amplia, voz gruesa y mirada amable, que se imponía por presencia. Pese a su aspecto más bien serio, que a algunos provocaba un cierto temor reverencial, poseía un corazón amable y una gran sensibilidad ante el sufrimiento y la muerte. Parco en palabras, se le escuchaba con respeto y atención, en la seguridad de que en su decir siempre había una enseñanza valiosa. Era el único a quien en la Cátedra se le llamaba «Profeson», en tanto que sus ayudantes, por connotados que fueran, eran únicamente «doctores». Era tal el prestigio de su Cátedra que los jóvenes estudiantes competían fuertemente por cursar sus estudios en ella, a la que ingresaban según sus méritos escolares. Estudioso incansable y ávido lector de la literatura médica, distribuía sus revistas entre sus ayudantes, marcando con sus nombres los artículos que les recomendaba leer a cada cual y, luego, las donaba a la biblioteca del hospital. Su espíritu tolerante se reflejaba en la diversidad de pensamientos que existía entre sus colaboradores, a quienes estimaba por sus capacidades y no por sus ideologías. Esto no impedía que con algunos de los más apasionados se tomara la licencia de hacerles bromas en público, con simpatía y humor. Tenía una actitud benevolente con los enfermos y los estudiantes, sin perjuicio de ser muy severo en exigir el cumplimiento de los deberes profesionales y escolares. Asombraba su habilidad en el diagnóstico clínico, en una época en que los recursos auxiliares eran mucho más escasos e imprecisos que hoy. Su talento diagnóstico brotaba de su capacidad de observación, de sus conocimientos y habilidades semiológicas y de su amplio dominio de la patolo-

\section{REFERENCIAS}

1. Acta de la Sesión Solemne Pública de 23 de abril de 1981 para rendir tributo al Doctor Hernán Alessandri Rodríguez. Boletín de la Academia de Medicina 1981; 22: 7-18.

2. EtcheverRy R. Homenaje al Profesor Dr. Hernán Alessandri Rodríguez (Discurso de Incorpora- gía, todo lo cual procesaba magistralmente su inteligencia privilegiada. Verlo sentado al lado de la cama del enfermo, conversándole con amabilidad y examinándolo delicadamente con sus grandes manos, nos traía a la memoria el clásico cuadro «The Doctor» de Sir Luke Fildes, una síntesis pictórica excepcionalmente bien lograda de la relación médico-paciente. Culminaban esos momentos ejemplares e inolvidables con una palabra de aliento al enfermo y de optimismo sobre el pronóstico de su enfermedad. Escéptico del efecto de los medicamentos que imponía la propaganda y la moda, con un dejo de ironía acostumbraba a decir a sus discípulos: hay que aprovechar los primeros seis meses en que ellos son efectivos», haciendo referencia así a su efecto placebo. Con gran sabiduría y sentido humanitario, cuando la medicina ya no tenía más que ofrecer a un paciente desahuciado, afirmaba: hay momentos en que la ciencia debe dar paso a la caridad».

Pero, el eminente clínico fue, además y sobre todo, un insigne universitario y educador, haciendo así honor a su condición de doctor, es decir, el maestro, el que enseña, instruye, el que hace aprender; y de médico: el que cura, alivia y cuida. Todo en él tenía una intención docente: su palabra, sus gestos, su actitud, su acencamiento a los enfermos, el ambiente que rodeaba su Cátedra, la organización asistencial de su Servicio. Penetrar los recintos del viejo hospital que albergaba su Cátedra, era impregnarse de un ambiente de sabiduná, de disciplina médica, de respeto y compasión por los enfermos, así como experimentar la presencia viva de una auténtica Universidad. Feliz ocasión la de hoy, para recordanlo con el cariño y la admiración que merece un médico y profesor universitanio excepcional, que le dio dignidad y prestigio a la medicina chilena, de lo que fueron testigos sus contemporáneos, en tanto que la historia de la medicina nacional lo ha acogido como una figura ejemplar e imperecedera para las nuevas generaciones de médicos.

ción 5 de agosto 1981). En: Boletín de la Academia de Medicina 1981; 22: 40-48.

3. Neghme A. Hernán Alessandri Rodríguez. Médico, Maestro, Académico y Universitario. Su vida y su obra. Editorial Universitaria. Santiago de Chile: 1982.

4. Gorc A. El Dr. Alessandri que yo conocí. Rev Méd Chile 1982; 110: 593-5. 